\title{
Kinematic Analysis and Dynamic Optimization Simulation of a Novel Unpowered Exoskeleton with Parallel Topology
}

\author{
Qiang Yan $\mathbb{D}^{1},{ }^{1}$ Jianjun Zhang $\mathbb{D}^{1},{ }^{1}$ Bin Li $\mathbb{D}^{2},{ }^{2}$ and Liang Zhou $\mathbb{D}^{1}$ \\ ${ }^{1}$ Hebei Key Laboratory for Parallel Equipment, Hebei University of Technology, Tianjin 300132, China \\ ${ }^{2}$ Tianjin Key Laboratory for Advanced Mechatronic System Design and Intelligent Control Tianjin University of Technology, \\ Tianjin 300384, China
}

Correspondence should be addressed to Jianjun Zhang; zhjjun96@139.com

Received 1 October 2018; Revised 23 November 2018; Accepted 19 June 2019; Published 9 July 2019

Academic Editor: Shahram Payandeh

Copyright (C) 2019 Qiang Yan et al. This is an open access article distributed under the Creative Commons Attribution License, which permits unrestricted use, distribution, and reproduction in any medium, provided the original work is properly cited.

\begin{abstract}
This paper studies the kinematic and dynamic analysis of a novel unpowered exoskeleton with topology. Firstly, the kinematics of the unpowered exoskeleton is analyzed by the derivation of the closed-loop position equation, and the forward position problems of the exoskeleton are obtained. Secondly, with the aim of doing some research in the dynamics, two of links for the exoskeleton are changed into flexible links. Some shapes concerning some parameters are acquired by simulation with fitting curve method. Thirdly, meanwhile, the dynamic model is built by using Lagrange method. Fourthly, the gait experiment is acquired with the aim of obtaining the law of the human joints. Fifthly, the dynamic model is verified by Adams software and the theoretical calculation. Meanwhile, an optimization is completed in the Adams software. The most reasonable spring stiffness is acquired. Finally, some conclusions are enumerated to show the properties of the mechanisms.
\end{abstract}

\section{Introduction}

Exoskeletons have been applied in various industrial fields these years. It was reported that the logistics in China is the largest all over the world. Meanwhile, load-carrying population leads to a serious problem; by the end of 2050, more than 140 million old people aged 60 or more will have great difficulty carrying loads, which is a huge increase in recent years [1]. In addition, considering various situations, such as the power the worker owns, working circumstance, and the limited tools [2], the number of working individuals with unpowered lower extremity limb is increasing year by year, which enormously reduces the burden of the workers. Considering the situation above, it was of significance for us to design an effective load-carrying tool, with the aim of helping workers not only get a certain degree of carrying, but also fully use the energy from the carriers. Currently, related papers showed that the existing lower limb load-carrying robot has mainly two forms, powered and unpowered lower extremity exoskeleton. Research in powered human exoskeleton starts from the late 1960s and numerous achievements have occurred in recent decades. The
Berkeley Lower Extremity Exoskeleton(BLEEX) was a powered exoskeleton, which can significantly enhance the ability of workers. BLEEX [3] with three DOFs was a novel structure, which applied to the commercial product which can assist person to walk [4]. The exoskeleton such as HAL (hybrid assistive limb) was presented in the University of Tsukuba. Its detailed structure is described in [5]. The RoboKnee which had one degree was proposed by Florida Institute for Human \& Machine Cognition and Robotics; the innovation point is that torque was applied across the knee to alleviate the user's quadriceps muscles. The RoboKnee made the wearer climb stairs and accomplish deep knee bends while carrying load on their back. IMAE (IHMC Mobility Assist Exoskeleton) was designed in Florida Institute for Human \& Machine Cognition; as for this exoskeleton, four links were undriven [6]. Researchers in Japan have designed an exoskeleton to help nurses transfer patients. With the aim of enhancing loadcarrying ability, the SJTU-EX is developed in Shanghai Jiao Tong University. In recent years, reducing the energy cost of walking with an unpowered device is a hot issue in the field of exoskeleton. It can lower the energy consumption and enhance the load-carrying ability of persons. Steven H. [7] 


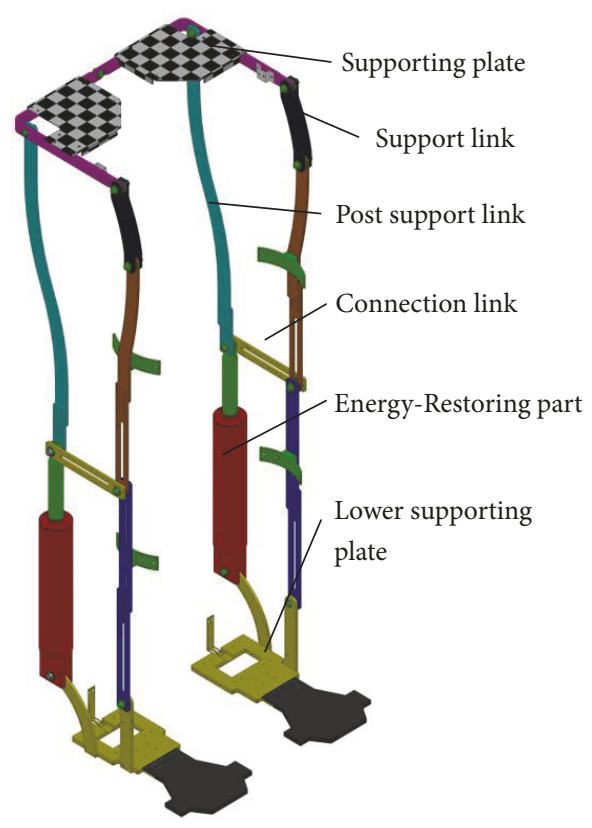

FIGURE 1: CAD model of the proposed unpowered exoskeleton.

designed an unpowered exoskeleton. Based on experiments, some conclusions were carried out. In 2016, Donghai et al. [8] presented the design and analysis of a passive body weight (BW)-support lower extremity exoskeleton (LEE) with compliant joints to relieve compressive load in the knee. The new design fully uses the energy of human muscle storage. Recently, the design, kinematic, and dynamic problems including position analysis, singularity analysis workspace, and force analysis of the exoskeleton have been studied by Cuan-Urquizo [9]. Article [10] presented a novel exoskeleton and analysis of the workspace of the swing leg. Meanwhile, the most reasonable spring stiffness was gotten by genetic algorithm. Compared with [10], the main contribution of this paper is to validate the forward dynamic equation of the mechanism by Adams software and theoretical deduction. At the same time, the consistency of human-machine coordination is verified by human joint experiments. Meanwhile, the influence factors of energy-storing device and the change of spring with time are analyzed. Besides these exoskeletons mentioned above, some other exoskeletons and parallel architectures can be found in the literature [10-22].

In this paper, a novel unpowered exoskeleton is described. This paper is designed as follows. Firstly, the structure characteristics and kinematics of this exoskeleton are analyzed by using closed vector method in Sections 2 and 3 , respectively. The dynamic performance of the mechanism for the exoskeleton is obtained based on the software of Adams in Section 4. In Section 5, using computer code programming and Adams software, the dynamic model is verified. Meanwhile, the deformation of the spring is analyzed. Lastly, Section 6 summarizes the full paper.

\section{The Description and Model of the Structure}

Human lower extremity limb consists of three parts: hip joints, knee joints, and ankle joints. Hips are ball-and-socket joints that can realize movements of flexion and extension, abduction and adduction, internal rotation, and external rotation. Knee joints have flexion, extension, internal rotation, and external rotation. Ankle joints also have three kinds of movements. The novel unpowered lower extremity limb exoskeleton (NNLELE) for this research is designed to make full use of the kinetic energy for a person who is walking. The eccentric task of the NNLELE is to help the carriers to prolong the working time and lower the consumption of the energy. It is well known for us that the main motion plane is sagittal plane. Therefore, the motion of the sagittal planes is taken completely into consideration due to cost, as this motion is easy to implement by NNLELE. To reduce costs, a modularized design was adopted for the NNLELE, as shown in Figure 1. The two legs of the robot consist of four kinematic branches: two rear support chains with two energy-storing devices, and two front anthropomorphic lower limbs which are designed by the stand motion of individuals. The detailed internal structure of energy-storing device for NNLELE is depicted in Figure 2. It comprises 12 parts; each part is illustrated in the figure. As depicted above, it is well known for us that the two legs both have the same kinematic characteristics. Therefore, we can take one leg as the study object to establish a kinematic model of the swing leg using closed vector circle method. The one side swing leg can be simplified to a closed four-degree mechanism with two planar linkages, as shown in Figure 3. Here, the workspace of NNLELE is restricted in the sagittal plane. The kinematic analysis of mechanism is an analysis of 


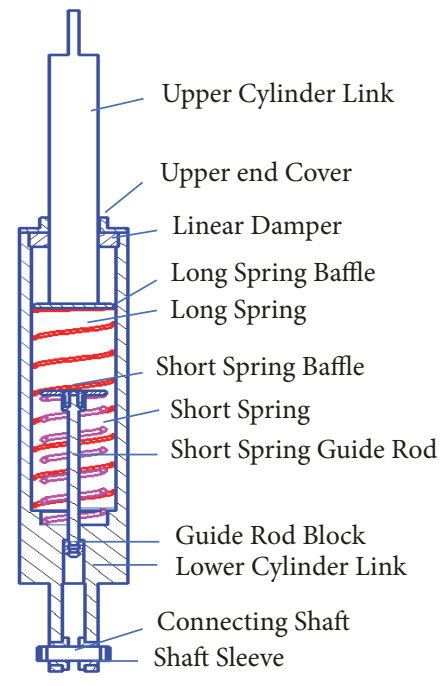

FIgURE 2: CAD model of the energy-storing device.

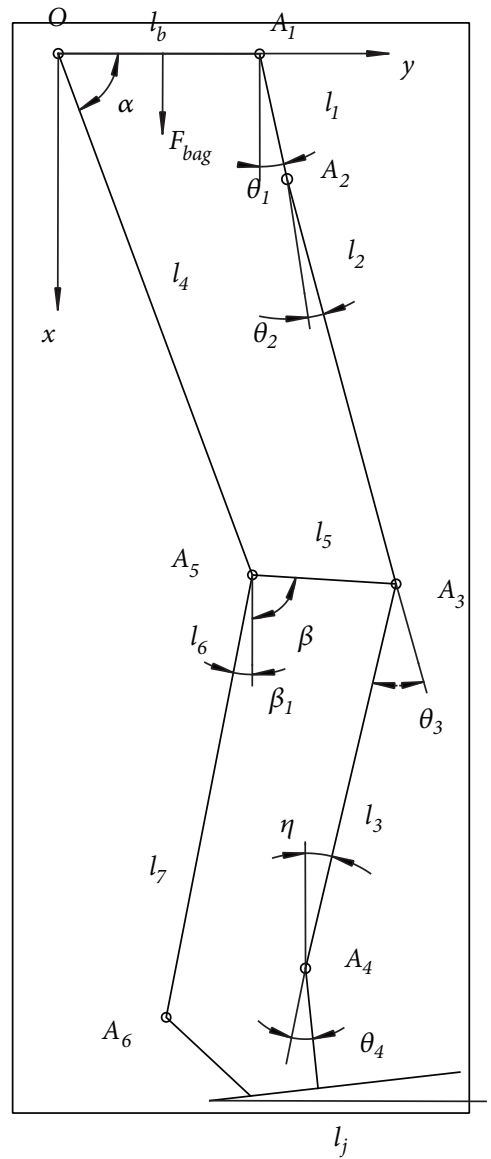

FIGURE 3: The schematic model of the swing leg.

the rigid body's position, velocity, and acceleration regardless of taking the force into account; it consists of forward and inverse kinematics. Forward kinematics is used to calculate the connecting rod position with the condition of the given rotation angle of each joint. Inverse kinematics is adapted to calculate the length of every link or the revolute angle of each joint by the given position of the end-effector. The swing leg has two branch chains: one is connected by $R-R-R-R$, and the other by $R-R-C S-R$. Here, $R$, CS represent the revolute joint, energy-containing device. For the proposed mechanism, the 
structure with parallel topology is just moving in the sagittal plane and the CS can restore the energy which comes from the kinetic energy of individuals.

Figures 1-3 of this article are taken from article [23].

\section{Forward Position Analysis of the Mechanism}

Set the coordinate system $O-x y$ attached to post-supporting with $O$ and link $O A_{1}$, with the $x$ axes being consistent with $O A_{1}, y$ axes being perpendicular to the $O A_{1}$, and $O$ being the origins. The signs $L_{b}, L_{1}, L_{2}, L_{3}, L_{1}, L_{4}$, and $L_{5}$ represent the length of the lines $O A_{1}, A_{1} A_{2}, A_{2} A_{3}, A_{3} A_{4}$, and $A_{6} A_{3}$,respectively. The angles are expressed in Figure 3 . It is important to note that $\beta$ is the angle between the vector $A_{6} A_{3}$ and the vertical direction and $\beta_{1}$ is that between the vector $A_{6} A_{5}$ and the vertical direction. The kinematics equation can be expressed by closed vector method.

$$
\begin{aligned}
& \overrightarrow{O A}_{3}=\overrightarrow{O A}_{1}+{\overrightarrow{A_{1} A_{2}}}+{\overrightarrow{A_{2} A_{3}}}^{\overrightarrow{O A}_{3}}=\overrightarrow{O A}_{6}+{\overrightarrow{A_{6} A_{3}}}
\end{aligned}
$$

The formulas can be rewritten as follows.

$$
\begin{aligned}
\left\|O A_{6} A_{3}\right\|_{x} & =l_{b}+l \times \sin \left(\theta_{1}\right)+l_{2} \times \sin \left(\theta_{2}+\theta_{1}\right) \\
\left\|O A_{1} A_{2} A_{3}\right\|_{x} & =l_{4} \times \cos (\alpha)+l_{5} \times \sin (\beta) \\
\left\|O A_{1} A_{2} A_{3}\right\|_{y} & =l_{4} \times \sin (\alpha)+l_{5} \times \cos (\beta) \\
\left\|O A_{6} A_{3}\right\|_{y} & =l_{1} \times \cos \left(\theta_{1}\right)+l_{2} \times \cos \left(\theta_{1}+\theta_{2}\right)
\end{aligned}
$$

However, it is significantly important for us to note that, according to Figure 3, the $L_{5}$ is a constant, so the constrain condition can be depicted as follows.

$$
\left\|A_{6} A_{3}\right\|=l_{5}
$$

From (2) to (8), the coordinates of the angle and knee can be ensured.

$$
\begin{aligned}
& \cos (\alpha)=\left(l_{b}+l_{1} \times \sin \left(\theta_{1}\right)+l_{2} \times \sin \left(\theta_{1}+\theta_{2}\right)\right) \frac{1}{l_{4}} \\
& \sin (\beta)=(A-B) \frac{1}{l_{5}}
\end{aligned}
$$

Here,

$$
\begin{aligned}
& A=l_{b}+l_{1} \times \sin \left(\theta_{1}\right)+l_{2} \times \sin \left(\theta_{1}+\theta_{2}\right) \\
& B=l_{1} \times \cos \left(\theta_{1}\right)+l_{2} \times \cos \left(\theta_{1}+\theta_{2}\right) .
\end{aligned}
$$

The pose of the knee is depicted as below

$$
\begin{aligned}
& x=l_{b}+l_{1} \times \sin \left(\theta_{1}\right)+l_{2} \times \sin \left(\theta_{1}+\theta_{2}\right) \\
& y=l_{1} \times \cos \left(\theta_{1}\right)+l_{2} \times \cos \left(\theta_{1}+\theta_{2}\right)
\end{aligned}
$$

so the coordinates of the angle in the sagittal plane can be described as follows.

$$
\begin{aligned}
x_{1}= & l_{b}+l_{1} \times \sin \left(\theta_{1}\right)+l_{2} \times \sin \left(\theta_{1}+\theta_{2}\right)+l_{3} \\
& \times \sin \left(\theta_{3}-\theta_{1}-\theta_{2}\right) \\
y_{1}= & l_{1} \times \cos \left(\theta_{1}\right)+l_{2} \times \cos \left(\theta_{1}+\theta_{2}\right)+l_{3} \\
& \times \cos \left(\theta_{3}-\theta_{1}-\theta_{2}\right)
\end{aligned}
$$

According to formulas (2), (3), (4), (5), (6), (7), (8), (9), (11), (12), (13), and (14), representing the designed ULLPE in the paper, the forward position analyses of the mechanism can be carried out straightly by analytical method; the location of the joints for the knee and ankle, which is extraordinarily fundamental for doing some research in workspace, can be acquired.

Here $\left(x_{1}, y_{1}\right)$ indicates the coordinate of the ankle joint and $(x, y)$ is the coordinate of the knee joint.

\section{Kinematic Simulation, Angles Parameters Analyses}

Here, with the condition of the formulas, the properties of some parameters are investigated. Some parameters are set as below:

$l_{b}=100 \mathrm{~mm}, l_{1}=133.42 \mathrm{~mm}, l_{2}=435.49 \mathrm{~mm}, l_{3}=410 \mathrm{~mm}$, $l_{4}=569.32 \mathrm{~mm}, l_{5}=425 \mathrm{~mm}, l_{6=}-20^{\circ} \leq \theta_{2} \leq 30^{\circ}, 0 \leq \theta_{3} \leq 30$, $0 \leq \theta_{4} \leq 30^{\circ}$.

However, in order to do some research in the new unpowered exoskeleton, the CAD model of the exoskeleton is imported in the Adams, the model of the novel exoskeleton can be shown in Figure 4 and the simulation of work is proposed as set in the following.

Here, the angles such as $\theta_{2}, \theta_{3}$, and $\theta_{4}$ stand for the rotating angles of waist, knee, and ankle. For better and clear understanding, we here use knee and ankle to represent the rotating angles.

With the help of the software Adams, the data concerning the values of the spring deformation for the CS and the angles is exported and dealt with by MATLAB with the fitting curve method. By simulation, Figures 5, 6, and 7, illustrate the relation among the spring deformation and every angle along with the change of time. Here, as we all known, the max and min values can be seen in Figure 4.

\section{The Dynamic Analysis of the Swing Leg on the Exoskeleton}

The accurate establishment of the dynamic model for the novel unpowered lower extremity exoskeleton is the precondition to realize the precise dynamic analysis. The forward kinematics for the unpowered exoskeleton has already been analyzed by closed vector method. Firstly, the period of motion of the lower limb is divided into two stages: oneleg support and two-leg support. The dynamic model of swing leg for the load-carrying robot system is established by Lagrange method. Secondly, through the real-time motion capture of human lower limb movement and the data analysis of its motion trajectory (including hip, knee, and ankle), the motion law of human joints during normal walking is 


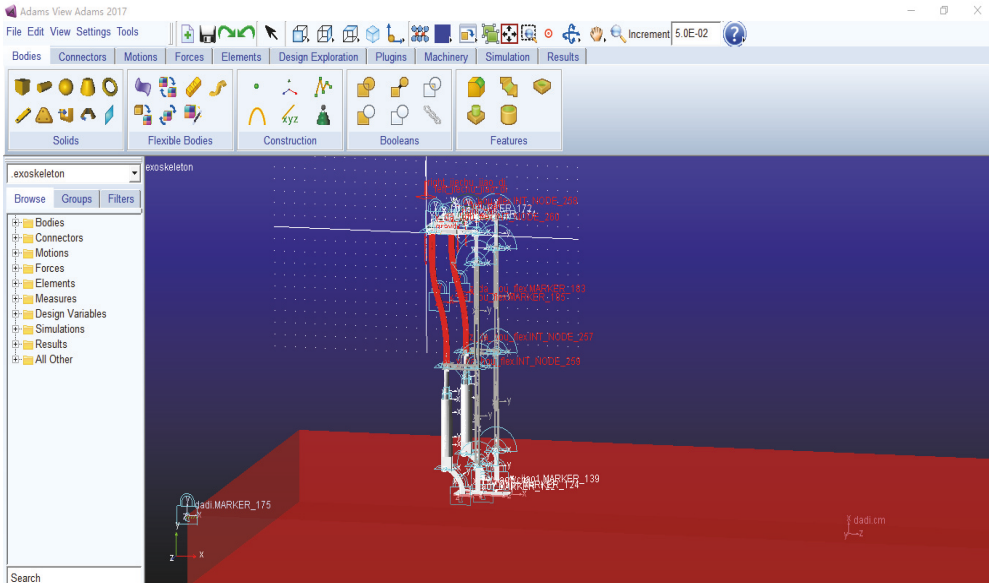

FIGURE 4: The three dimensions of the novel exoskeleton in Adams.

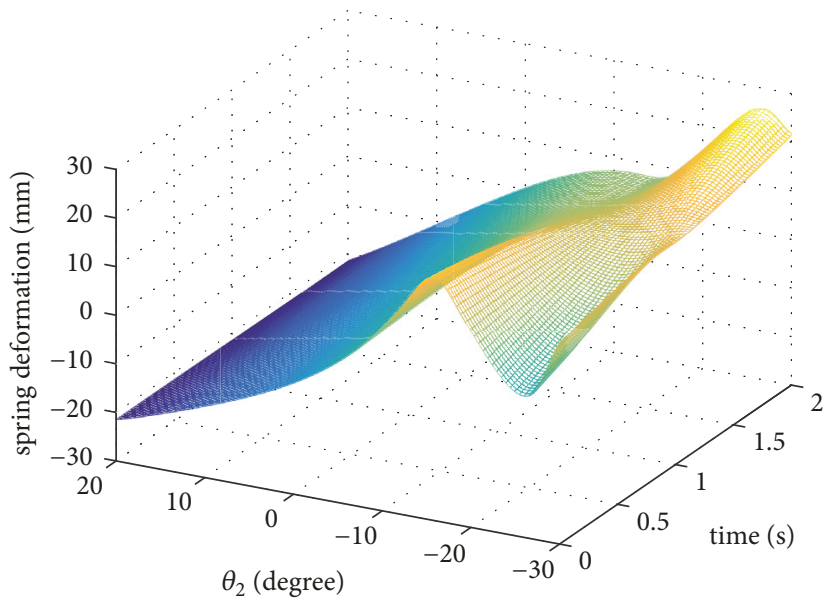

FIGURE 5: The relation between knee and $\alpha$.

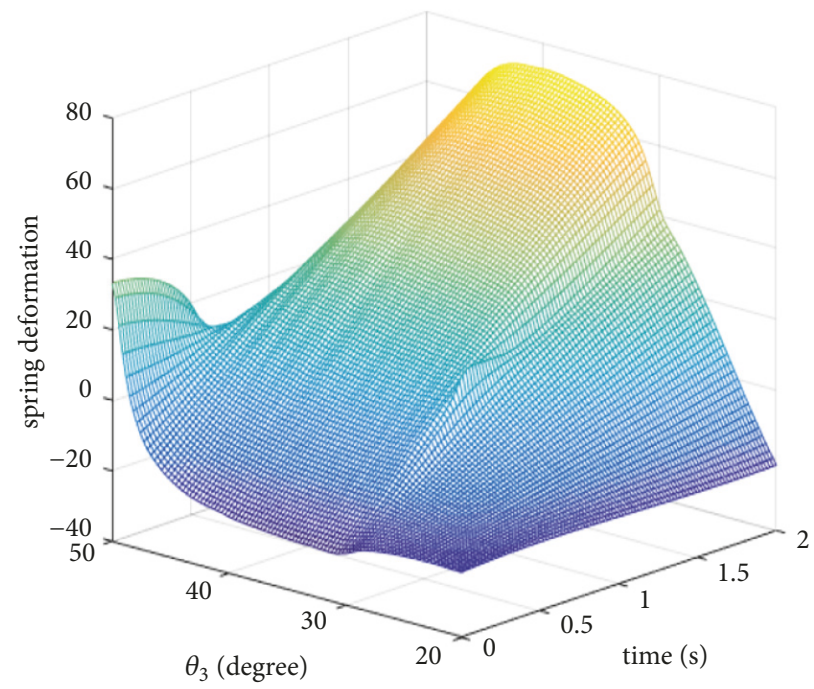

FIgURE 6: The relation between knee and $\beta$. 


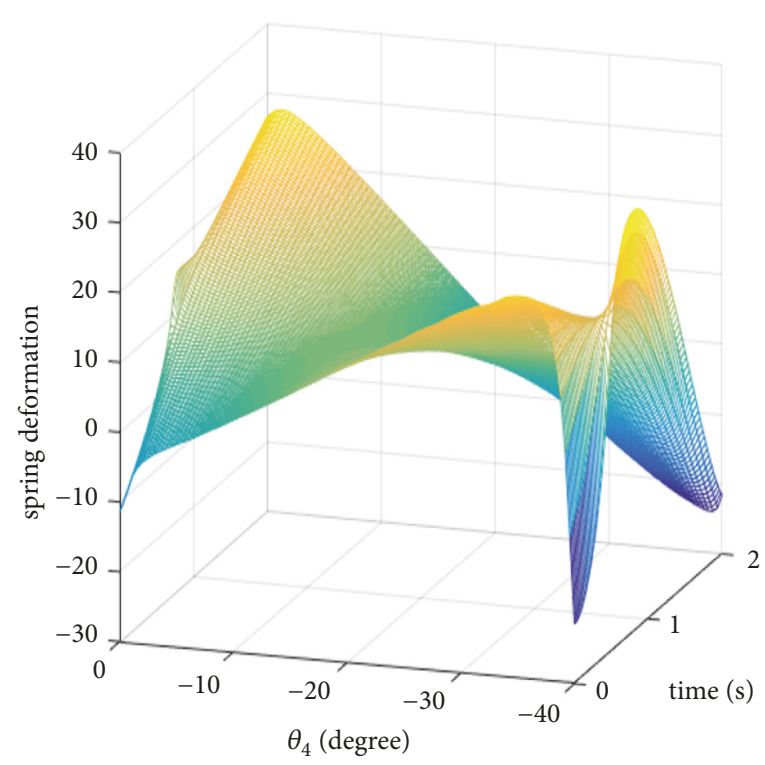

FIgURE 7: The relation between knee and $\beta_{1}$.

obtained with fitting method. Thirdly, putting the motion law into the dynamic equations, the driving torque including is obtained. Fourthly, the $3 \mathrm{D}$ simplified model of robot is established by SolidWorks and the dynamic simulation is carried out by importing Adams software. The accuracy of theoretical derivation is verified. The theoretical basis is provided for further research of the unpowered lower extremity exoskeleton and some conclusions are drawn.

5.1. The Accurate Establishment of the Dynamic Model on Exoskeleton. As demonstrated in Figure 2, the structure of the swing leg can be seen clearly. In this part, in order to clearly express the dynamic formula, the $\alpha$ is instead of $\theta 5$ and the $\beta$ is the $\theta 6$. The mass center $\left(x_{m i}, y_{m i}\right)$ of each link can be easily gotten, and the detailed description can be listed as follows.

$$
\begin{aligned}
x_{m 1}= & \frac{l_{b}}{2}, \\
y_{m 1}= & 0 \\
x_{m 2}= & l_{b}+l_{1} \sin \theta_{1}, \\
y_{m 2}= & \frac{l_{1} \cos \theta}{2} \\
x_{m 3}= & l_{b}+l_{1} \sin \theta_{1}+l_{2} \sin \left(\theta_{1}+\theta_{2}\right) \\
y_{m 3}= & l_{1} \cos \theta_{1}+\frac{l_{2} \cos \left(\theta_{1}+\theta_{2}\right)}{2} \\
x_{m 4}= & l_{b}+l_{1} \sin \theta_{1}+l_{2} \sin \left(\theta_{1}+\theta_{2}\right) \\
& +l_{3} \sin \left(\theta_{3}-\theta_{2}-\theta_{1}\right) \\
y_{m 4}= & l_{1} \cos \theta_{1}+l_{2} \cos \left(\theta_{1}+\theta_{2}\right) \\
& +\frac{l_{3} \cos \left(\theta_{3}-\theta_{2}-\theta_{1}\right)}{2}
\end{aligned}
$$

$$
\begin{aligned}
& x_{m 5}=\frac{l_{4} \cos \theta_{5}}{2}, \\
& y_{m 5}=\frac{l_{4} \sin \theta_{5}}{2} \\
& x_{m 6}=\frac{l_{5} \sin \theta_{6}}{2}+l_{4} \cos \theta_{5}, \\
& y_{m 6}=\frac{l_{5} \cos \theta_{6}}{2}+l_{4} \sin \theta_{5} \\
& x_{m 7}=l_{4} \cos \theta_{5}-\frac{\left(l_{6}+l_{7}\right) \sin \theta_{7}}{2} \\
& y_{m 7}=l_{4} \sin \theta_{5}+\frac{\left(l_{6}+l_{7}\right) \cos \theta_{7}}{2} \\
& x_{m 8}=\left(x_{m 4}+x_{m 6}\right) \\
& y_{m 8}=\left(y_{m 4}+y_{m 6}\right)
\end{aligned}
$$

The elastic potential energy consists of two parts, one is from the gravity potential energy, and the other is from the energystoring device. Firstly, the energy-storing device potential energy can be expressed as follows.

$$
\begin{aligned}
U_{1} & =\frac{k x^{2}}{2} \\
x & =\left\|A_{5} A_{6}\right\|-\left(l_{6}+l_{7}-h\right) \\
\left\|A_{5} A_{6}\right\|^{2} & =\left(x_{5}-x_{6}\right)^{2}+\left(y_{5}-y_{6}\right)^{2}
\end{aligned}
$$

Here, $U_{1}$ is the potential energy of the energy-storing device. $h$ is the compressing length resulting from the force. $x_{5}, x_{6}$, $y_{5}, y_{6}$ stand for the coordinates of the points $A_{5}, A 6 ; \mathrm{x}$ is the compressed length of spring. 
Thus, according to the Lagrange method, the formula can be listed as follows.

$$
L=E_{K 1}-E_{P 1}
$$

Here, $E_{p 1}$ stands for the overall gravity potential energy from each link, and $E_{k l}$ is the kinematic energy of swing leg.

The overall potential energy can be described as follows.

$$
\begin{aligned}
E_{p 1} & =U_{1}+U_{2} \\
U_{2} & =\sum_{i=1}^{8} m_{i} g y_{m i}
\end{aligned}
$$

Here, $U_{2}$ is the overall gravity potential energy, $g$ is the gravity acceleration velocity, $m_{i}$ is the mass of each link for the swing leg, and $I_{i}$ is the moment of inertia of each link.

$$
\begin{aligned}
E_{k 1} & =\sum_{i=1}^{8} \frac{1}{2 m_{i}\left(\left(\dot{x}_{m i}\right)^{2}+\left(\dot{y}_{m i}\right)^{2}\right)}+\sum_{i=1}^{8} \frac{1}{2 I_{i}\left(\dot{\theta}_{i}\right)^{2}}, \\
I_{i} & =\frac{1}{3 m_{i} l_{i}^{2}}
\end{aligned}
$$

Therefore, on the basis of the above deduction related to the dynamic model of the swing leg, the driving torque can be expressed as below.

$$
T_{i}=\frac{d}{d t}\left(\frac{\partial L}{\partial \dot{\theta}_{i}}\right)-\frac{\partial L}{\partial \theta_{i}}+\frac{\partial E}{\partial \theta_{i}}
$$

5.2. The Workspace of Deformation $x$ on Spring. In order to do some research in the dynamics and the deformation of the spring, some parameters can be set as follows: here, the angles are set to $-40^{\circ} \leq \theta_{1} \leq 40^{\circ},-40^{\circ} \leq \theta_{2} \leq 40^{\circ},-50 \leq \theta_{3} \leq 50,-20$ $\leq \theta_{4} \leq 20^{\circ}$.

According to (16), the workspace of the $\mathrm{x}$ is analyzed. The relationship among the parameters is depicted in Figure 8.

From Figure 8, the relationship can be obtained and the arrangement can be gotten.

5.3. Human Gait Data Acquisition and Dynamic Simulation. For the lower extremity exoskeleton robot, the movement gait according to the physiological habits of the human body and the realization of the assist function under the action of the control system are important issues for studying the lower extremity exoskeleton robot. The movement gait of the human body has the characteristics of periodicity, repeatability, and symmetry. Therefore, it is only necessary to obtain the motion data of the human body during one cycle of normal walking to obtain the motion law of the human body during the whole movement.

5.4. Human Gait Data Acquisition. The laboratory is equipped with a three-dimensional motion capture and analysis system. It mainly consists of FASTCAM SA-X29 (depicted in Figure 9) which is produced by the company of Photron. Using Photron's FASTCAM SA-X2 camera to capture the person's walking process, we can easily obtain the data of the joint of hip, knee, and ankle. It accurately captures subtle changes in the human lower limb movement and restores each true change of each picture. As described in Figures 9 and 10, the walker wears clothes with special colors attached. The special color parts are the sole, the bottom of the calf, the top of the calf, the bottom of the thigh, the top of the thigh, and the waist.

The specific process of human gait information collection is shown in Figure 10. The experimenter wears the test suit and pastes the identification points on the left and right ankles, the left and right knee joints, the left and right hip joints, and other important features. The high-speed camera is used to capture the identification of the experimenter. Selecting the indoor $10 \mathrm{~m}$ straight line distance as the data acquisition distance, the experimenter walks about $1.5 \mathrm{~m} / \mathrm{s}$ and sets the sampling period to $0.02 \mathrm{~s}$. Before each collection, the experimenter stands upright and calibrates the origin of 12 high-speed cameras in the lab to reduce experimental errors due to human factors.

5.5. The Motion Law Acquisition of Human's Movement. The experimenter repeats the motion according to the predetermined linear trajectory. The high-speed cameras record the pictures of the recognition point in real time and stores it in the computer. The position coordinates of the Cartesian space are transformed into discrete joint angle changes by the cosine function. The data is imported into MATLAB with the aim of curve fitting.

$$
\begin{aligned}
f(t)= & a_{0}+\sum_{i=1}^{n} a_{i} * \sin (i * w * t)+\sum_{i=1}^{n} b_{i} \\
& * \cos (i * w * t)
\end{aligned}
$$

Here, $a_{i}, b_{i}$ are the coefficients of the $\mathrm{i}$-th order trigonometric function; $w$ is the angular frequency.

The 3D motion capture and analysis system and acquisition process are shown in Figure 10. The cosine theorem data is restored to the joint angle value, and the curve fitting is performed to obtain the angle change law.

As depicted in Figures 11(a), 11(b), and 11(c), according to (21), the motion law of swing leg including hip, knee, and ankle joints of walker is obtained.

5.6. Calculation and Contraction on the Driving Torque for the Exoskeleton. Putting the body's joint law into the formulas (15)-(20), the driving torque (expressed by $\tau 1 \sim \tau 4$ ) can be gotten. Meanwhile, the driving torque changes with the changes of time as shown in Figures 12(a), 12(b), 12(c), and 12(d). At the same time, the body's joint law was imported into the Adams, and the driving torque which is expressed by M1 M4 can be gotten. The data from the Adams is imported and the law is drawn, which is depicted in Figures 12(a), 12(b), $12(\mathrm{c})$, and $12(\mathrm{~d})$ concerning the relationship between the driving torque and the time. The contraction is verifying the rightness of the forward dynamic model. The parameters can be defined as follows; for other parameters, refer to Section 4 .

$$
\begin{aligned}
& m_{b}=4 \mathrm{~kg}, \\
& m_{1}=3 \mathrm{~kg},
\end{aligned}
$$




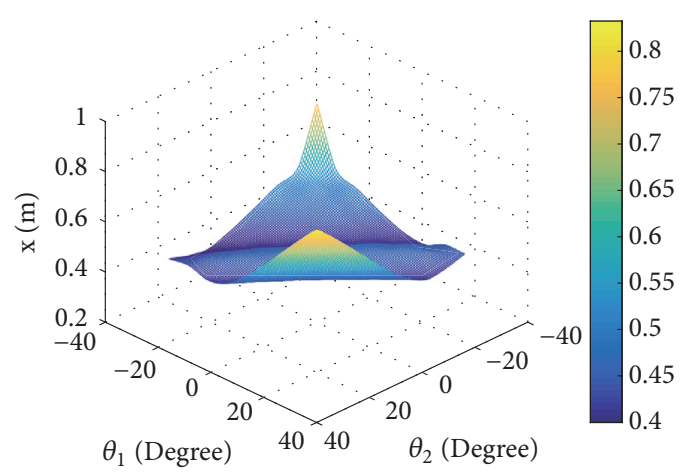

(a)

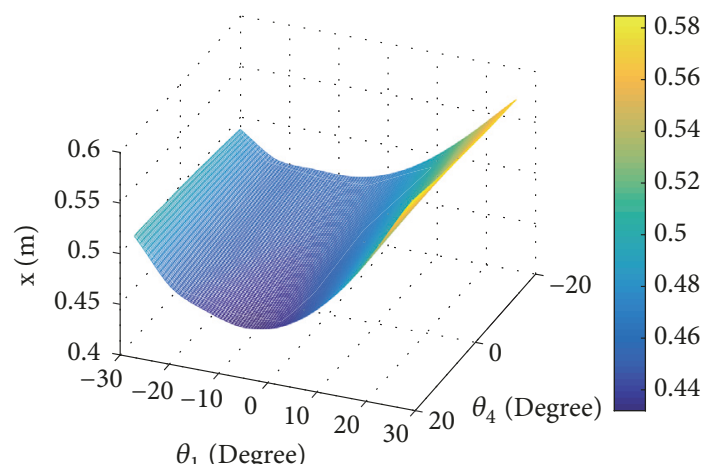

(c)

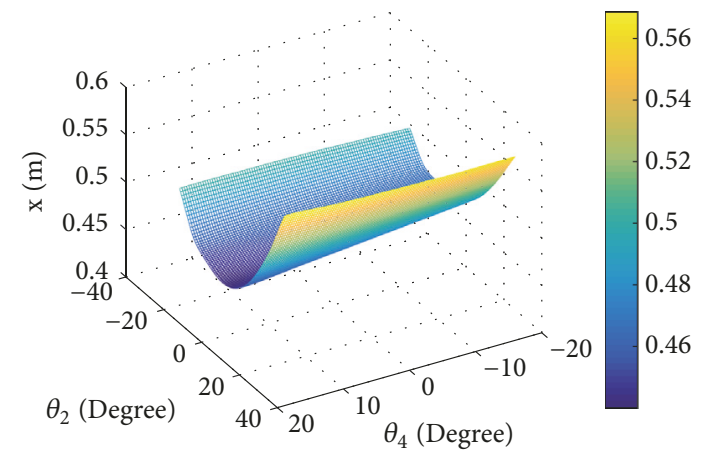

(e)

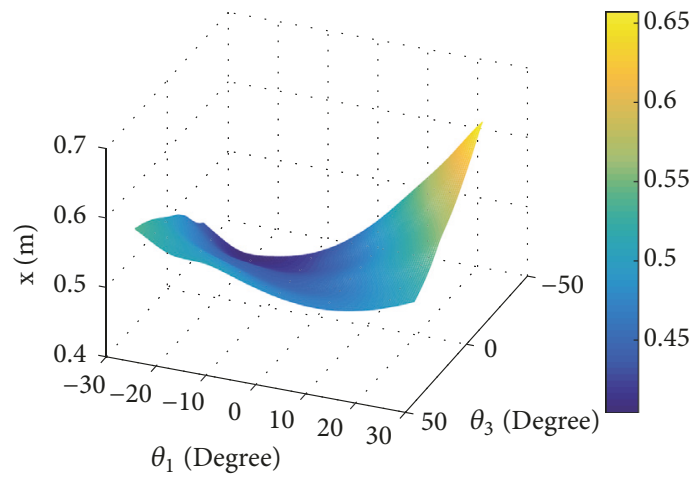

(b)

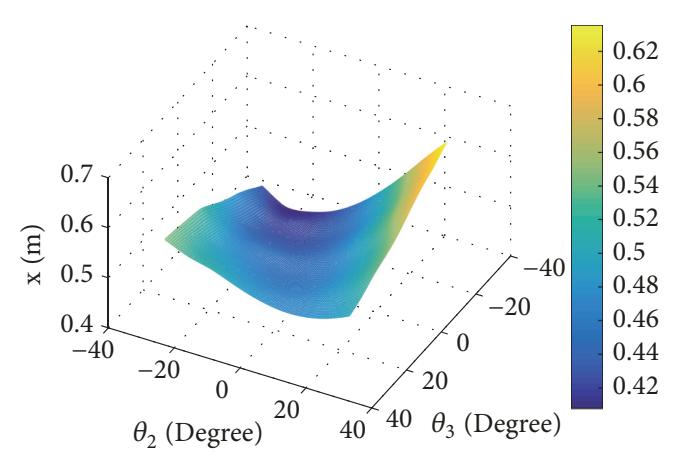

(d)

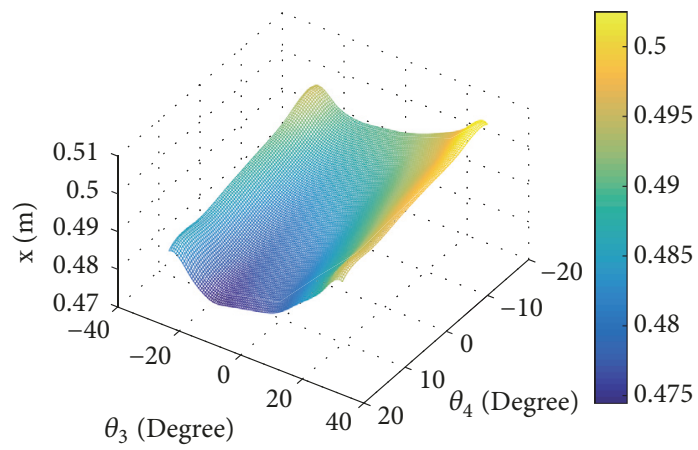

(f)

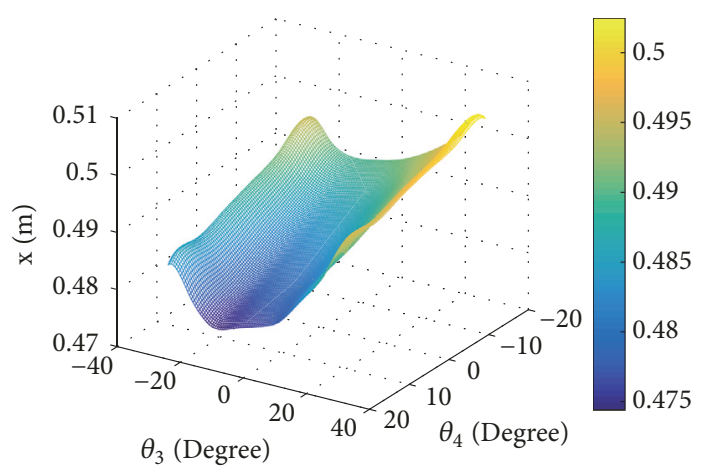

(g)

FIGURE 8: The workspace of the deformation on the spring. 


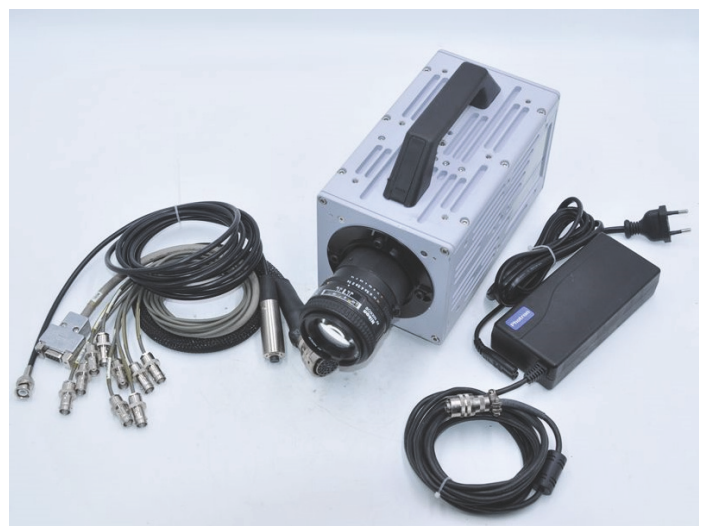

Figure 9: The camera of FASTCAM SA-X29.

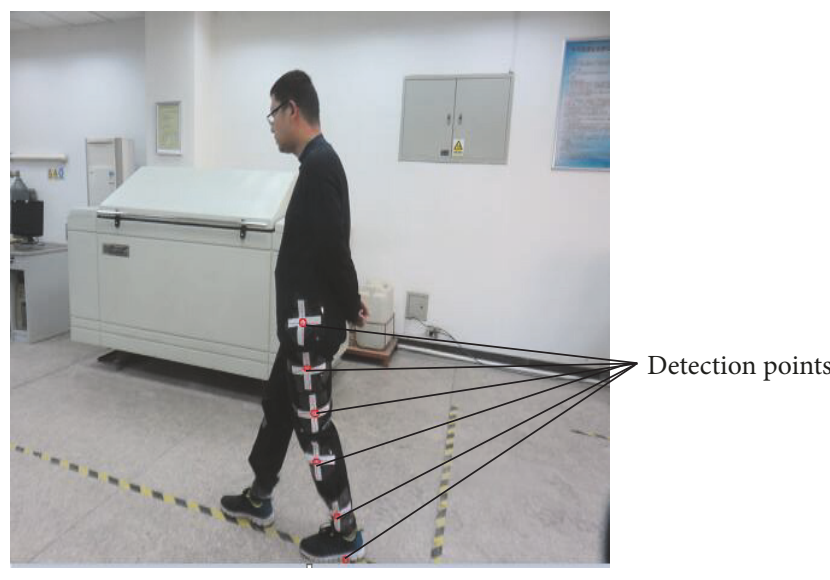

FIGURE 10: Data acquisition location of a person.

$$
\begin{aligned}
m_{2} & =9 \mathrm{~kg}, \\
m_{3} & =8 \mathrm{~kg}, \\
m_{4} & =10 \mathrm{~kg}, \\
m_{5} & =3 \mathrm{~kg}, \\
m_{6} & =4 \mathrm{~kg}, \\
m_{7} & =6 \mathrm{~kg}, \\
m_{8} & =3 \mathrm{~kg}, \\
l_{7} & =300 \mathrm{~mm}, \\
l_{j} & =l_{8}=300 \mathrm{~mm}, \\
k & =2, \\
l_{k t} & =450
\end{aligned}
$$

According to Figure 12, we can see the change law of each driving torque (from the theoretical analysis and the Adams model) exerted on the waist, hip, knee, and the ankle joints. The range of driving torque for the waist joint, the hip, the knee, and the ankle has some errors. The comparison found that there is an error between the theoretical calculation and the Adams software simulation results, but the difference is not big, and the trend is basically the same. The main reason for the difference between theoretical analysis and simulation results is that in the calculation of dynamic theory, the robot is regarded as the model under ideal state, ignoring the influence of ground friction and assembly error, when performing virtual prototype simulation. There are also some errors in the stiffness and friction settings and the spline interpolation function fitted by the experimental motion data, which results in a difference between the theoretical calculation and the simulation results.

5.7. The Relationship between Spring Force and the Compressed Spring Length. The spring force can be written as follows.

$$
F=k * x
$$

Here, $\mathrm{F}$ stands for the spring force and $\mathrm{x}$ is the compressed spring length. On the basis of establishing the dynamics and gait experiment concerning the swinging leg, the relationship between the spring force and the compressed spring length of the energy storage element can be expressed according to formula (23), so the variation law of the force-dependent variable and time is as shown in the Figure 13. As shown in Figure 13, we can clearly see the relationship among spring force, spring compression, and time.

5.8. Simulation Conditions, Parameter Settings, and Optimization Results. Here, while optimizing the spring stiffness, it is needed to follow some constraining conditions:

(1) The motion properties with regard to the unpowered exoskeleton should be set rightly such as the various motion pairs and forces defined in the software of Adams.

(2) The model in the Adams should be simulated normally and the dynamic analysis makes the overall structure effective.

Because the optimization is fully completed in Adams software, the objective function and the design variable are 


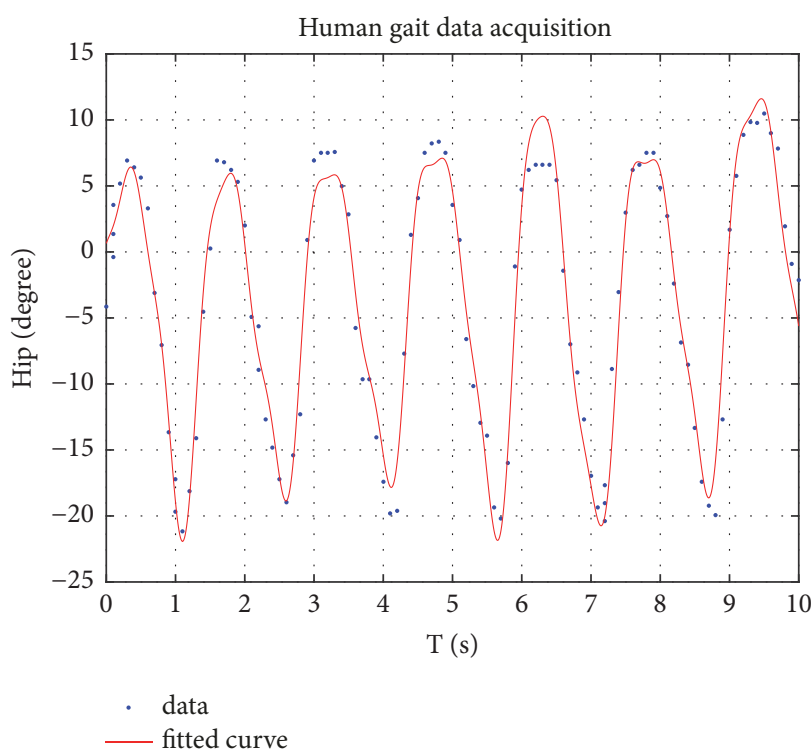

(a)

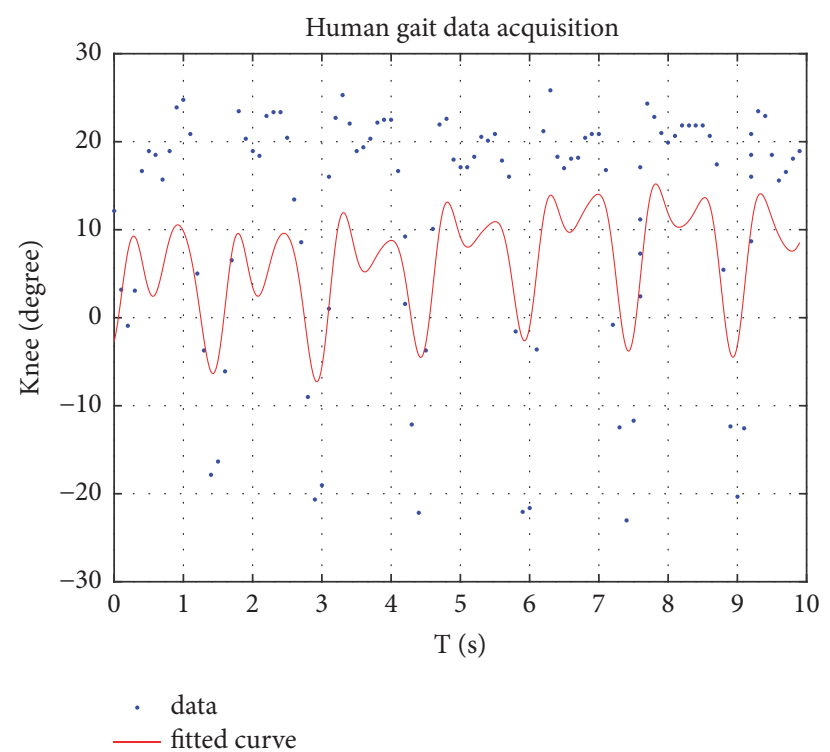

(b)

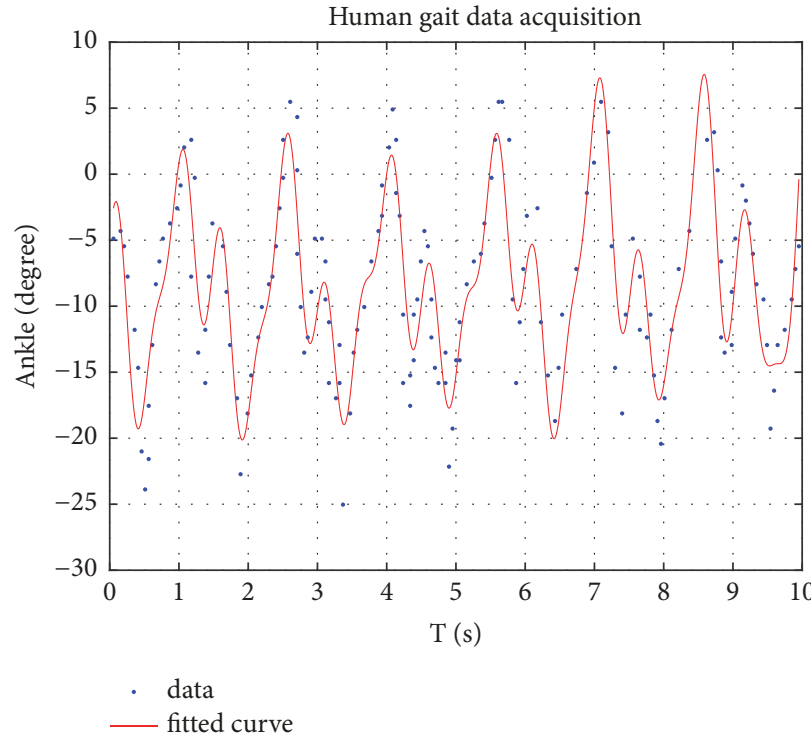

(c)

FIGURE 11: The movement law on each human joint for the swing leg.

shown in Figure 4, as well as the force cloud diagram of the flexible rod.

During a gait cycle, the torque/force exerted on the flexible link $l_{4}$ can be seen clearly. The middle and the end of link $\mathrm{l}_{4}$ bear a big force. In order to enhance the performance of the exoskeleton, it is necessary to optimize the spring. By simulation, the reasonable spring stiffness is 5.7995 .

\section{Conclusions}

In this paper, a novel unpowered exoskeleton is proposed. From the analysis mentioned above, some conclusions can be draw as follows. Firstly, the structure of the exoskeleton is described. Secondly, the forward position analysis is derived by closed vector method. Thirdly, during a gait cycle, the relation among angle parameters is gotten as shown in Figures 5-7. Fourthly, in order to explore some dynamic properties of the exoskeleton, two links of the exoskeleton are changed into flexible links. Meanwhile, some data is exported and dealt with using fitting curve method in the MATLAB software. Meanwhile, the dynamic model is built by using Lagrange method. Fifthly, the gait experiment is gotten with aim of obtaining the law of the human joints and the workspace of the deformation on the spring is analyzed. Finally, the dynamic model is verified by Adams software and the theoretical calculation. An optimization is completed in the Adams software. The most reasonable spring stiffness is acquired. Based on the kinematics analysis of the mechanism, dynamic optimization analysis, the related investigation of the mechanism will be explored in future work. 

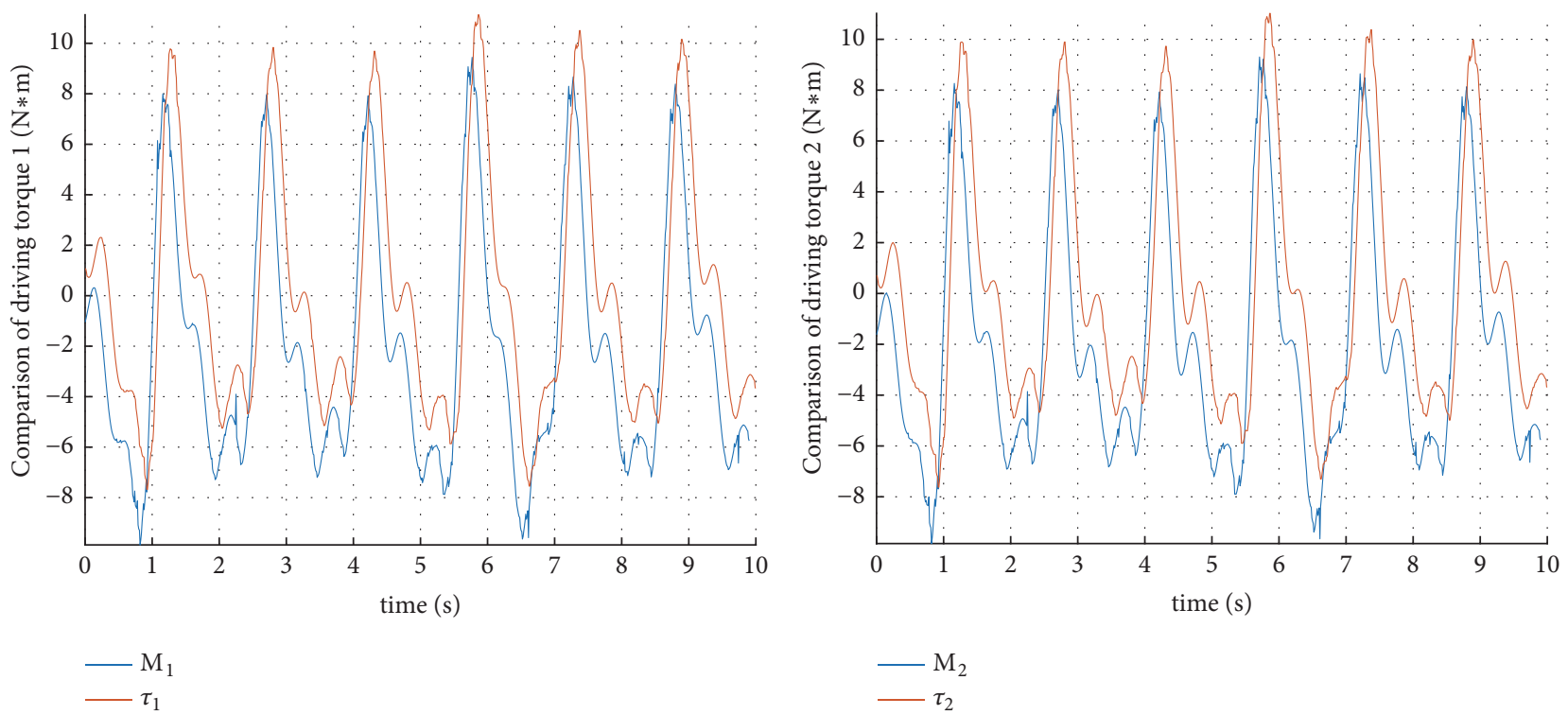

(a)

(b)
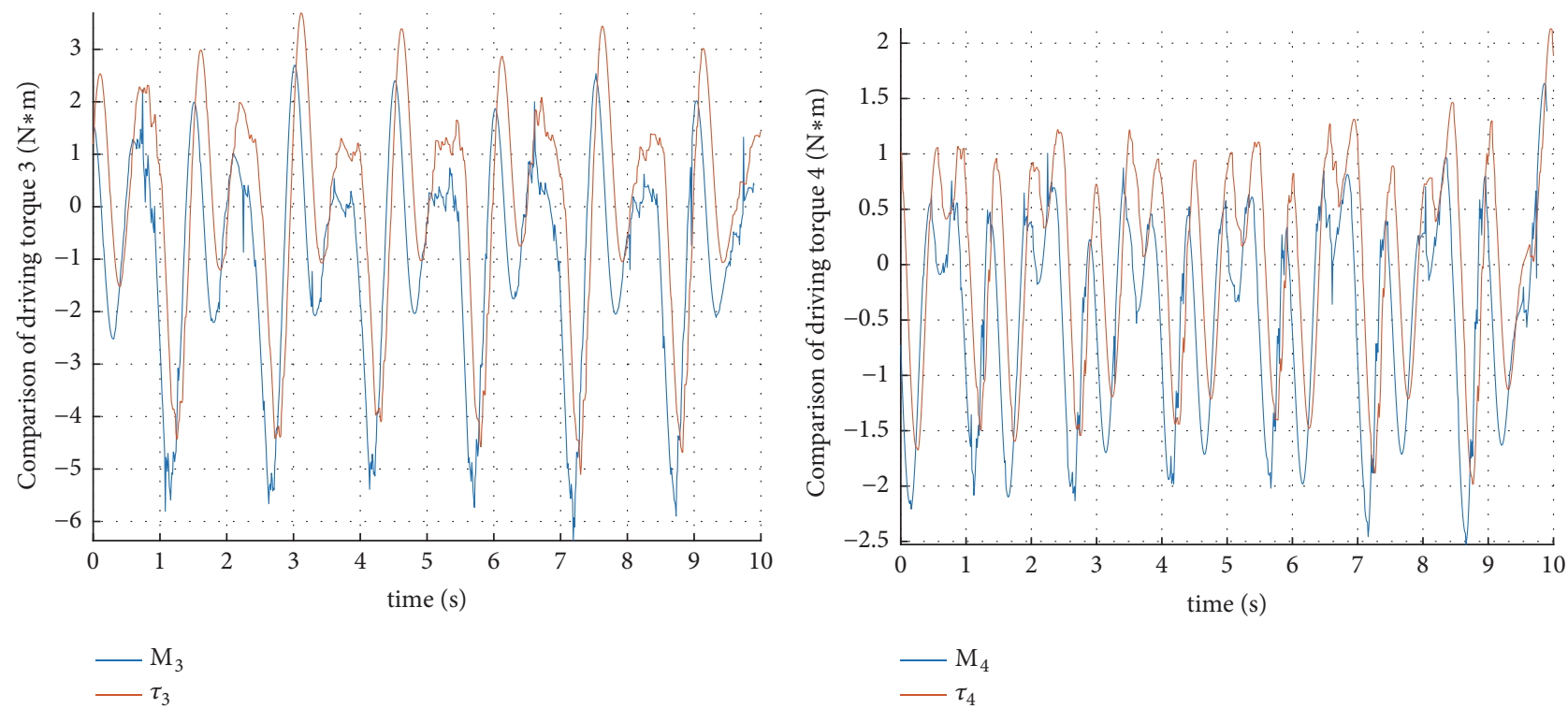

(c)

(d)

FIGURE 12: The variation of diving torque on each joint.

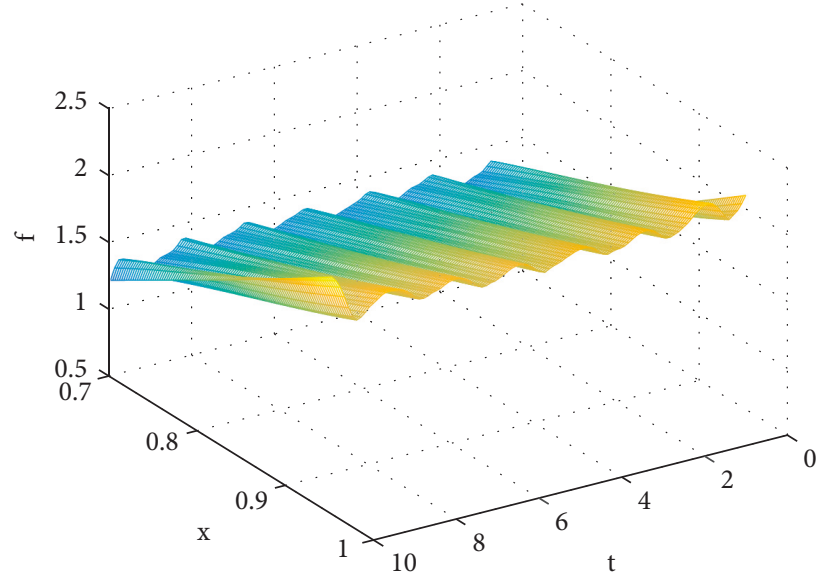

FIGURE 13: The change of spring force. 


\section{Data Availability}

The data used to support the findings of this study are available from the corresponding author upon request.

\section{Conflicts of Interest}

The authors declare that they have no conflicts of interest.

\section{Acknowledgments}

The National Natural Science Foundation of China (NSFC) (Grant No. 51175144) and the Tianjin Natural Science Foundation of China (TNSFC) (Grant No. 17JCZDJC40200) supported this work.

\section{References}

[1] J. C. Qian, Y. D. Chen, K. Q. Rao, and D. Meng, "Analysis and suggestions of disability men in China," Chinese Journal of Health Statistics, vol. 29, no. 1, pp. 6-9, 2012.

[2] B. Zhou, Q. L. Sun, D. C. Gui, X. D. Li, and J. W. Guo, "A study on the prognosis of patients with stroke during early rehabilitation," Chinese Journal of Rehabilitation Medicine, vol. 15 , no. 5, pp. 269-271, 2000.

[3] A. Chu, H. Kazerooni, and A. Zoss, "On the biomimetic design of the Berkeley lower extremity exoskeleton (BLEEX)," in Proceedings of the 2005 ICRA on Robotics and Automation, 2005.

[4] H. Kazerooni and R. Steger, "The Berkeley lower extremity exoskeleton," Journal of Dynamic Systems, Measurement, and Control, vol. 128, p. 14, 2006.

[5] H. Kawamoto, S. Lee, S. Kanbe et al., "Power assist method for HAL-3 using EMG-based feedback controller," in Proceedings of the 2003 IEEE International Conference on Systems, Man and Cybernetics, pp. 1648-1653, Washington DC, USA, 2003.

[6] J. E. Pratt, B. T. Krupp, C. J. Morse et al., "The RoboKnee: an exoskeleton for enhancing strength and endurance during walking," IEEE, vol. 3, pp. 2430-2435, 2004.

[7] H. K. Kwa, J. H. Noorden, M. Missel et al., "Development of the ihmc mobility assist exoskeleton," in Proceedings of the 2009 IEEE International Conference on Robotics and Automation (ICRA '09), pp. 2556-2562, Japan, 2009.

[8] S. H. Collins, M. B. Wiggin, and G. S. Sawicki, "Reducing the energy cost of human walking using an unpowered exoskeleton," Nature, vol. 522, pp. 212-215, 2015.

[9] D. Wang, K.-M. Lee, and J. Ji, "A Passive Gait-Based WeightSupport Lower Extremity Exoskeleton with Compliant Joints," IEEE Transactions on Robotics, vol. 32, no. 4, pp. 933-942, 2016.

[10] A. Chaker, A. Mlika, M. A. Laribi et al., "Accuracy analysis of non-overconstrained spherical parallel manipulators," European Journal of Mechanics - A/Solids, vol. 47, pp. 362-372, 2014.

[11] Q. Yan, B. Li, Y. Li, and X. Zhao, "Comparative Study of Two 2RPU\&1-SPR Parallel Manipulators," in Proceedings of the 2016 12th World Congress on Intelligent Control and Automation (WCICA '16), pp. 1975-1980, Guilin, China, 2016.

[12] Q. Yan, B. Li, Y. Li, and X. Zhao, "Comparative stiffness analysis of two over-constrained manipulators," in Proceedings of the 2016 IEEE International Conference on Advanced Robotics and Mechatronics, (ICARM '16), pp. 225-230, Macau, China, 2016.
[13] Q. Yan, B. Li, Y. Li, and X. Zhao, "Kinematics Comparative Study of Two Over-Constrained Parallel Manipulators," Mathematical Problems in Engineering, vol. 2016, Article ID 5091405, 12 pages, 2016.

[14] D. Wang, K.-M. Lee, and J. Ji, "A Passive Gait-Based WeightSupport Lower Extremity Exoskeleton with Compliant Joints," IEEE Transactions on Robotics, vol. 32, no. 4, 2016.

[15] X. J. Liu, J. Wang, and G. Pritschow, "A new family of spatial 3DoF fully-parallel manipulators with high rotational capability," Mechanism \& Machine Theory, vol. 40, no. 4, pp. 475-494, 2005.

[16] H. Khakpour, L. Birglen, and S.-A. Tahan, "Synthesis of differentially driven planar cable parallel manipulators," IEEE Transactions on Robotics, vol. 30, no. 3, pp. 619-630, 2014.

[17] Q. Zhao, J. Mei, T. Song, and S. Liu, "Pressure angle in parallel mechanisms: from planar to spatial," Transactions of Tianjin University, vol. 22, no. 5, pp. 411-418, 2016.

[18] L. M. Zhang, J. P. Mei, X. M. Zhao et al., "Dimensional synthesis of the Delta robot using transmission angle constraints dimensional synthesis of the Delta robot using transmission angle constraints," Robotica, vol. 30, no. 3, pp. 343-349, 2012.

[19] D. Zeng, Y. Hou, Z. Huang, and W. Lu, “Type synthesis and characteristic analysis of a family of 2-DOF rotational decoupled parallel mechanisms," Chinese Journal of Mechanical Engineering, vol. 22, no. 6, pp. 833-840, 2009.

[20] M. A. Hosseini, H.-R. M. Daniali, and H. D. Taghirad, "Dexterous workspace optimization of a Tricept parallel manipulator," Advanced Robotics, vol. 25, no. 13-14, pp. 1697-1712, 2011.

[21] P. Araujo-Gómez, V. Mata, M. Díaz-Rodríguez, A. Valera, and A. Page, "Design and kinematic analysis of a novel 3UPS/RPU parallel kinematic mechanism with 2T2R motion for knee diagnosis and rehabilitation tasks," Journal of Mechanisms and Robotics, 2017.

[22] Y. Miao, F. Gao, and D. Pan, "Prototype design and size optimization of a hybrid lower extremity exoskeleton with a scissor mechanism for load-carrying augmentation," Proceedings of the Institution of Mechanical Engineers, Part C: Journal of Mechanical Engineering Science, vol. 229, no. 1, pp. 155-167, 2015.

[23] Q. Yan, J. Zhang, and K. Qi, "Structure design and kinematics analysis of a novel unpowered load-carrying lower extremity exoskeleton with parallel topology," Mathematical Problems in Engineering, vol. 2018, Article ID 4128520, 10 pages, 2018. 


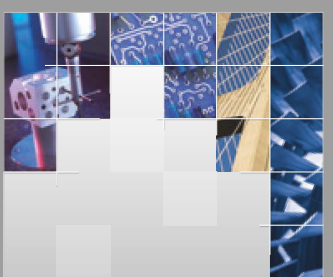

\section{Enfincering}
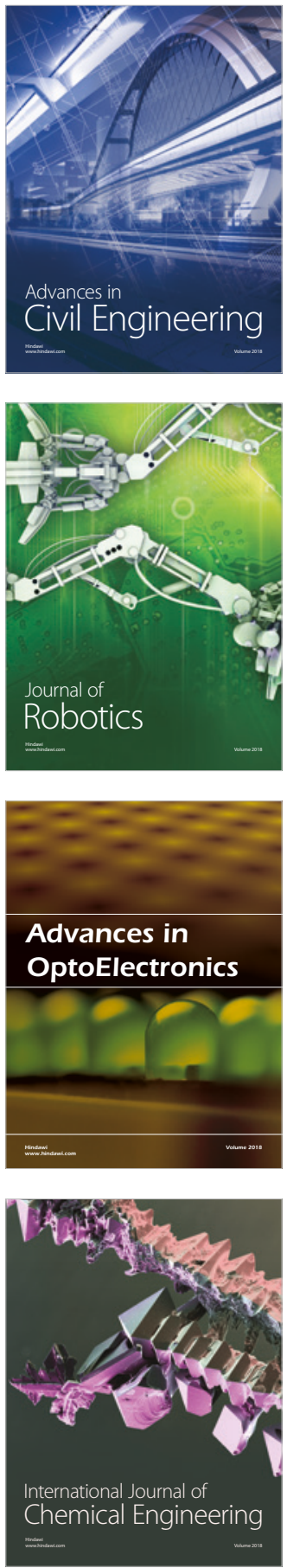

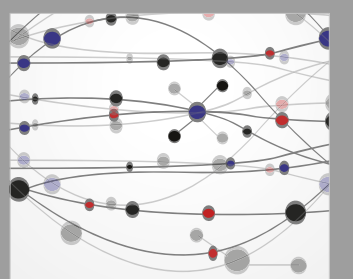

\section{Rotating \\ Machinery}

The Scientific World Journal

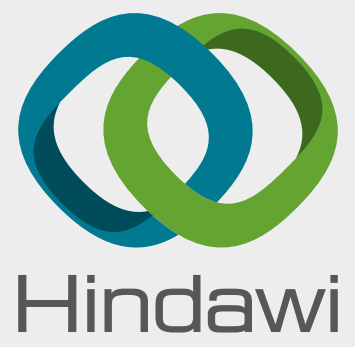

Submit your manuscripts at

www.hindawi.com
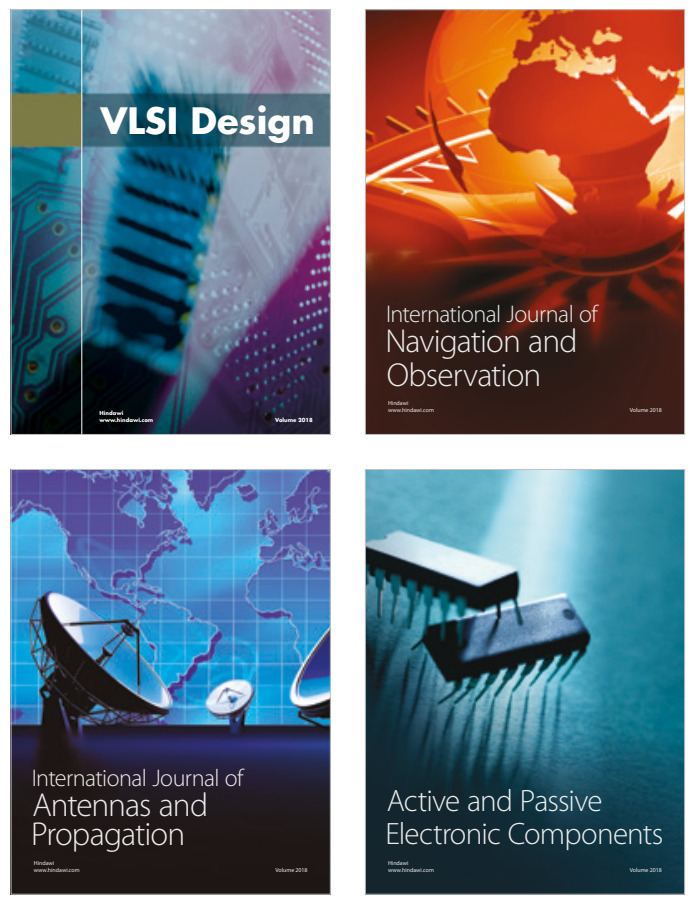
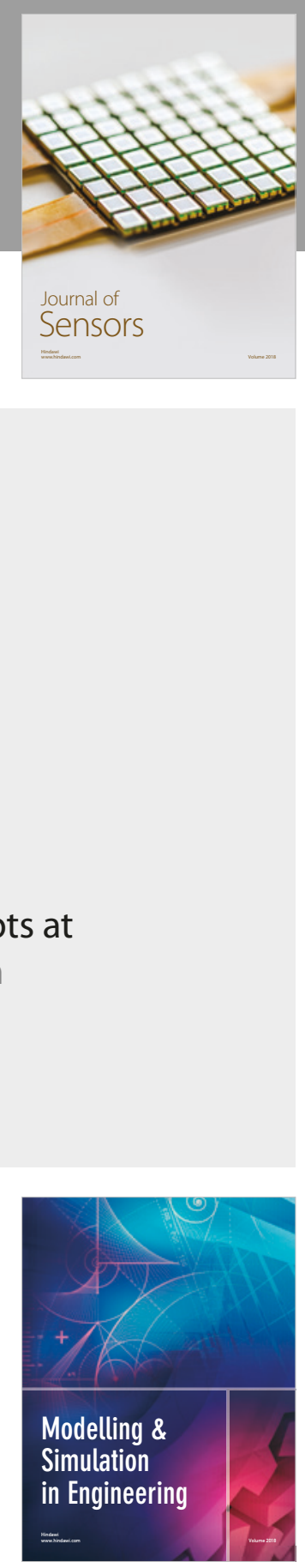

\section{Advances \\ Multimedia}
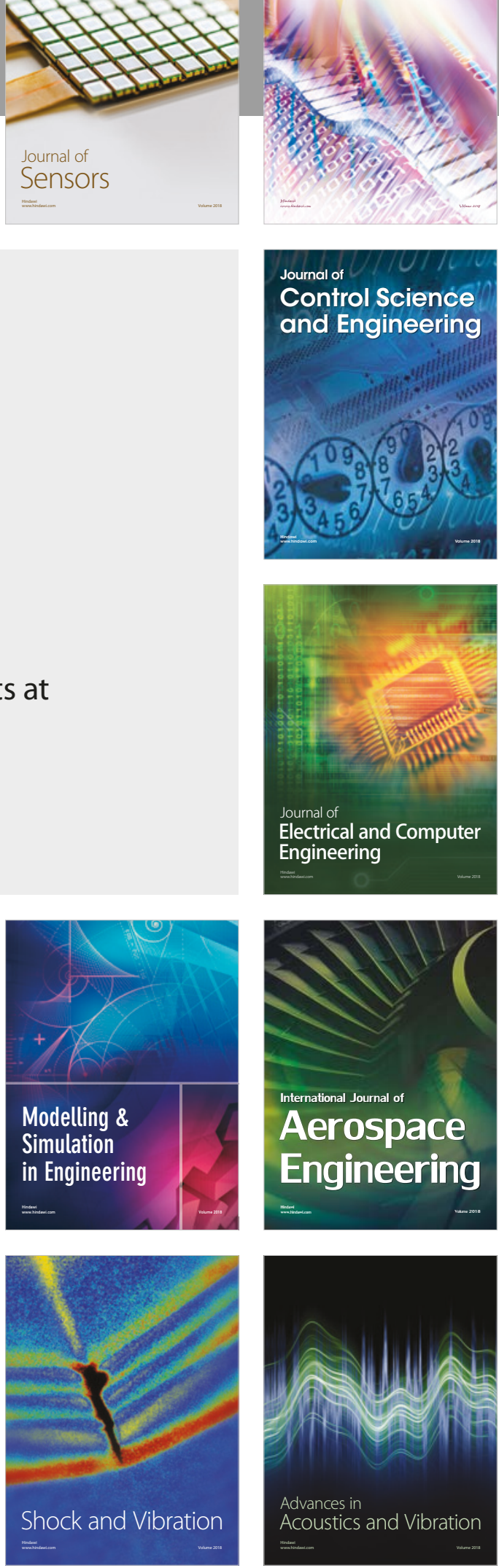